Article

\title{
Strong Tolerance and Strong Universality of Interval Eigenvectors in a Max-Łukasiewicz Algebra
}

\author{
Martin Gavalec ${ }^{1, * \mathbb{D}}$, Zuzana Němcová ${ }^{1}$ and Ján Plavka ${ }^{2}$ \\ 1 Faculty of Informatics and Management, University of Hradec Králové, 50003 Hradec Králové, \\ Czech Republic; zuzana.nemcova@uhk.cz \\ 2 Faculty of Electrical Engineering and Informatics, Technical University of Košice, 04200 Košice, Slovakia; \\ jan.plavka@tuke.sk \\ * Correspondence: martin.gavalec@uhk.cz; Tel.: +420-493-332-248
}

Received: 9 August 2020; Accepted: 2 September 2020; Published: 4 September 2020

\begin{abstract}
The Łukasiewicz conjunction (sometimes also considered to be a logic of absolute comparison), which is used in multivalued logic and in fuzzy set theory, is one of the most important t-norms. In combination with the binary operation 'maximum', the Łukasiewicz t-norm forms the basis for the so-called max-Łuk algebra, with applications to the investigation of systems working in discrete steps (discrete events systems; DES, in short). Similar algebras describing the work of DES's are based on other pairs of operations, such as max-min algebra, max-plus algebra, or max- $T$ algebra (with a given t-norm, $T$ ). The investigation of the steady states in a DES leads to the study of the eigenvectors of the transition matrix in the corresponding max-algebra. In real systems, the input values are usually taken to be in some interval. Various types of interval eigenvectors of interval matrices in max-min and max-plus algebras have been described. This paper is oriented to the investigation of strong, strongly tolerable, and strongly universal interval eigenvectors in a max-Łuk algebra. The main method used in this paper is based on max- $€$ linear combinations of matrices and vectors. Necessary and sufficient conditions for the recognition of strong, strongly tolerable, and strongly universal eigenvectors have been found. The theoretical results are illustrated by numerical examples.
\end{abstract}

Keywords: max-Łukasiewicz algebra; interval matrix; interval eigenvector; strong interval eigenvector

MSC: $90 \mathrm{C} 15$

\section{Introduction}

A max-Łukasiewicz algebra (max- $\succeq u k$ algebra, in short), is one of the so-called max- $T$ fuzzy algebras, which are defined for various triangular norms.

A max- $T$ fuzzy algebra contains values in the unit interval $\mathcal{I}=\langle 0,1\rangle$ and uses the binary operation of maximum and one of the triangular norms, $T$, instead of the conventional operations of addition and multiplication. Thus, by a max- $T$ fuzzy algebra we understand a triplet $\left(\mathcal{I}, \oplus_{,} \otimes_{T}\right)$, where $\mathcal{I}$ is the interval $\langle 0,1\rangle$ and $\oplus=\max , \otimes_{T}=T$ are binary operations on $\mathcal{I}$. The symbol $\mathcal{I}(m, n)$, respectively, $\mathcal{I}(n)$, denotes the set of all matrices (respectively, vectors) of the given dimensions over $\mathcal{I}$. The operations $\oplus, \otimes_{T}$ are extended to matrices and vectors in the standard way. The linear ordering on $\mathcal{I}$ induces partial orderings on $\mathcal{I}(m, n)$ and $\mathcal{I}(n)$.

The triangular norms (t-norms, in short) were introduced in [1], in the context of probabilistic metric spaces. The t-norms are interpreted as the conjunction in multi-valued fuzzy logics, or as the intersection of fuzzy sets. These functions are used in many fields, such as statistics and game theory, information and data fusion, decision making support, risk management, and probability 
theory. The t-norms (and the corresponding t-conorms) play an important role in fuzzy set theory. Many t-norms can be found in [2]).

The Łukasiewicz norm is often considered to be a logic of absolute (or metric) comparison. The Łukasiewicz conjunction is defined by

$$
x \otimes_{L} y=\max \{x+y-1,0\} .
$$

The simplest norm is the Gödel norm, and the conjunction is defined as the minimum of the entries: the truth degrees of the constituents. Gödel logic is considered to be a logic of relative comparison.

$$
x \otimes_{G} y=\min (x, y)
$$

In the particular case when $T=\min$ is the Gödel t-norm, we get an important max-min algebra which has useful applications to optimization and scheduling problems. Max-min algebras belong to the so-called tropical mathematics, with a wide scope of applications and interesting contributions to mathematical theory. Several monographs [3-6] and collections of papers [7-13] have dealt with tropical mathematics and related problems.

Tropical algebras can be naturally used for the study of systems working in discrete time (DES). The state of the system at time $t$ is described by a vector $x(t)$. The transitions of the system from one state to another are described by the transition matrix $A$. The next state $x(t+1)$ is obtained by multiplying the transition matrix and the state vector; in matrix notation we write $A \otimes x(t)=x(t+1)$. When a DES reaches a steady state, after some time of operation, then the state vectors of the steady states are eigenvectors of $A$. In any tropical algebra, the eigenproblem for a given matrix $A \in \mathcal{I}(n, n)$ consists of finding an eigenvalue $\lambda \in \mathcal{I}$ and an eigenvector $x \in \mathcal{I}(n)$ fulfilling $A \otimes x=\lambda \otimes x$.

The eigenproblem in tropical algebra has been described in many papers, see [14]. Interesting results describing the structure of the eigenspace and several algorithms for computing the largest eigenvector of a given matrix have been published, for example, in $[15,16]$. The eigenvectors in a max- $T$ algebra, for various triangular norms $T$, are useful in fuzzy set theory. Such eigenvectors have been studied in [17-19]. The eigenvalues and eigenvectors are interesting characteristics of the DES in fuzzy algebras. The eigenspace structures for the drastic and t-norm have been studied in $[18,19]$. Finally, [17] describes the case of Łukasiewicz fuzzy algebra.

\section{Strong Types of Interval Eigenvectors in Max-Łuk Algebras}

The investigation in this paper will be started by a simple numerical example.

Example 1. (Numerical illustration: Steady state vector).

Assume

$$
A=\left(\begin{array}{ccccc}
0.5 & 0.4 & 0.1 & 0.1 & 0.5 \\
0.8 & 0.8 & 0.2 & 0.1 & 0.7 \\
0.6 & 0.6 & 0.6 & 0.2 & 0 \\
0.4 & 0.5 & 0.5 & 0.2 & 0.4 \\
0.3 & 0.1 & 0.4 & 0 & 0.8
\end{array}\right), \quad x=\left(\begin{array}{c}
0.5 \\
0.7 \\
0.5 \\
0.4 \\
0.8
\end{array}\right), \quad \lambda=0.8
$$

Then

$$
A \otimes_{L} x=\left(\begin{array}{c}
0.3 \\
0.5 \\
0.3 \\
0.2 \\
0.6
\end{array}\right)=0.8 \otimes_{L} x
$$


That is, $x$ is a max- - uk eigenvector of $A$ with the eigenvalue $\lambda=0.8$.

In practical applications, the matrix entries usually are not exact numbers, but are contained in some intervals. Interval arithmetic is an efficient way to represent matrix operations on a computer. Similarly, matrices and vectors with interval coefficients are studied in a max-Łuk algebra (or a max-min algebra, or some other tropical algebra), see $[9,17,20-23]$. The classification of various types of the interval eigenvectors in a max-min algebra has been investigated in $[24,25]$.

Let $n$ be a given natural number. We define $N=\{1,2, \ldots, n\}$. Similarly to [21,25-27], we define the interval matrix with bounds $\underline{A}, \bar{A} \in \mathcal{I}(n, n)$ and the interval vector with bounds $\underline{x}, \bar{x} \in \mathcal{I}(n)$ as

$$
[\underline{A}, \bar{A}]=\{A \in \mathcal{I}(n, n) ; \underline{A} \leq A \leq \bar{A}\}, \quad[\underline{x}, \bar{x}]=\{x \in \mathcal{I}(n) ; \underline{x} \leq x \leq \bar{x}\} .
$$

Let us assume that an interval matrix $\mathbf{A}=[\underline{A}, \bar{A}]$ and an interval vector $\mathbf{X}=[\underline{x}, \bar{x}]$ have been fixed. The interval max- $€$ uk eigenproblem for $\mathbf{A}$ and $\mathbf{X}$ aims at recognizing whether $A \otimes_{L} x=\lambda \otimes_{L} x$ holds true for $A \in \mathbf{A}, x \in \mathbf{X}, \lambda \in \mathcal{I}$, with suitable quantifiers (e.g., for all $A \in \mathbf{A}$, for some $A \in \mathbf{A}$, for all $x \in \mathbf{X}$, for some $x \in \mathbf{X}$ ) and their various combinations. Various types of interval max- $€$ uk eigenvectors are defined, using various choices of quantifiers and their order (see [25] for the further classification types).

Definition 1. Assume that an interval matrix $\mathbf{A}$ and an interval vector $\mathbf{X}$ are given. Then, $\mathbf{X}$ is called:

- $\quad A$ strong max-Łuk eigenvector of $\mathbf{A}$

if $(\exists \lambda \in \mathcal{I})(\forall A \in \mathbf{A})(\forall x \in \mathbf{X})\left[A \otimes_{L} x=\lambda \otimes_{L} x\right]$;

- $\quad A$ strongly tolerable max-Łuk eigenvector of $\mathbf{A}$

if $(\exists \lambda \in \mathcal{I})(\exists A \in \mathbf{A})(\forall x \in \mathbf{X})\left[A \otimes_{L} x=\lambda \otimes_{L} x\right]$;

- $\quad A$ strongly universal max-Łuk eigenvector of $\mathbf{A}$ if $(\exists \lambda \in \mathcal{I})(\exists x \in \mathbf{X})(\forall A \in \mathbf{A})\left[A \otimes_{L} x=\lambda \otimes_{L} x\right]$.

Remark 1. In general, an interval vector $\mathbf{X}$ is called a tolerable max-Euk eigenvector of $\mathbf{A}$ if there is an eigenvalue $\lambda \in \mathcal{I}$ such that every $x \in \mathbf{X}$ preserves the state vector up to a multiple by $\lambda$, for some $A \in \mathbf{A}$ (in other words: $A$ tolerates $x$ with eigenvalue $\lambda$ ).

In the case when there is one common tolerating matrix $A \in \mathbf{A}$ for all of the vectors $x \in \mathbf{X}$, the interval vector $\mathbf{X}$ is called strongly tolerable. Otherwise, the tolerating matrix $A$ depends on $x$. If also the eigenvalue $\lambda$ depends on $x$, then the interval eigenvector $\mathbf{X}$ is usually called weakly tolerable.

Remark 2. Similarly, an interval vector $\mathbf{X}$ is called a universal max- $€$ uk eigenvector of $\mathbf{A}$ if there is an eigenvalue $\lambda \in \mathcal{I}$ such that for every $A \in \mathbf{A}$, some $x \in \mathbf{X}$ preserves the state vector up to a multiple by $\lambda$, (in other words: $x$ is universal for $A$ with eigenvalue $\lambda$ ).

In the case when there is one common universal vector $x \in \mathbf{X}$ for all matrices $A \in \mathbf{A}$, the interval vector $\mathbf{X}$, as well as the common universal $x$, are called strongly universal. Otherwise, the universal vector $x$ depends on $A$. If also the eigenvalue $\lambda$ depends on $A$, then the interval eigenvector $\mathbf{X}$ is usually called weakly universal.

In this paper, we study in more detail the strong max-Łuk interval eigenvectors, the strongly tolerable and strongly universal max-Łuk interval eigenvectors (the remaining types of max- $€$ uk interval eigenvector are not considered here). Necessary and sufficient conditions are described for recognizing whether a given interval vector is a strong (strongly tolerable, strongly universal) eigenvector of a given interval matrix in a max-Łuk algebra. The results are illustrated by numerical examples. 


\section{Strong Interval Eigenvectors in a Max-Łuk Algebra}

In this section, we assume that an interval matrix $\mathbf{A}=[\underline{A}, \bar{A}]$ and an interval vector $\mathbf{X}=[\underline{x}, \bar{x}]$ are given. For each pair $i, j \in N$, define $\tilde{A}^{(i j)} \in \mathcal{I}(n, n)$ and $\tilde{x}^{(i)} \in \mathcal{I}(n)$ by putting for every $k, l \in N$,

$$
\tilde{a}_{k l}^{(i j)}=\left\{\begin{array}{ll}
\bar{a}_{i j}, & \text { for } k=i, l=j \\
\underline{a}_{k l}, & \text { otherwise }
\end{array}, \quad \tilde{x}_{k}^{(i)}=\left\{\begin{array}{ll}
\bar{x}_{i,} & \text { for } k=i \\
\underline{x}_{k}, & \text { otherwise }
\end{array} .\right.\right.
$$

It is shown in the following lemma that every $A \in \mathbf{A}$ can be written as a max-Łuk linear combination of generators $\tilde{A}^{(i j)}$ with $i, j \in N$. Similarly, every $x \in \mathbf{X}$ is equal to a max-Łuk linear combination of generators $\tilde{x}^{(i)}$ with $i \in N$.

Lemma 1. Let $x \in \mathcal{I}(n)$ and $A \in \mathcal{I}(n, n)$. Then,

(i) $\quad x \in \mathbf{X}$ if and only if $x=\bigoplus_{i \in N} \beta_{i} \otimes_{L} \tilde{x}^{(i)}$ for some $\beta_{i} \in \mathcal{I}$ with $\underline{x}_{i}-\bar{x}_{i}+1 \leq \beta_{i} \leq 1$,

(ii) $\quad A \in \mathbf{A}$ if and only if $A=\bigoplus_{i, j \in N} \alpha_{i j} \otimes_{L} \tilde{A}^{(i j)}$ for some $\alpha_{i j} \in \mathcal{I}$ with $\underline{a}_{i j}-\bar{a}_{i j}+1 \leq \alpha_{i j} \leq 1$.

Proof. For the proof of statement (i), assume that $x \in \mathrm{X}$ : that is, $\underline{x}_{i} \leq x_{i} \leq \bar{x}_{i}$ for every $i \in N$. Put $\beta_{i}=x_{i}-\bar{x}_{i}+1$ for each $i \in N$. It is easy to see that the $\beta_{i}$ 's satisfy the inequalities in assertion (i). Moreover, for every $j \in N$

$$
\begin{aligned}
\left(\bigoplus_{i \in N} \beta_{i} \otimes_{L} \tilde{x}^{(i)}\right)_{j} & =\bigoplus_{i \in N}\left(\left(x_{i}-\bar{x}_{i}+1\right) \otimes_{L} \tilde{x}^{(i)}\right)_{j} \\
& =\left(\left(x_{j}-\bar{x}_{j}+1\right) \otimes_{L} \tilde{x}_{j}^{(j)}\right) \oplus \bigoplus_{i \in N \backslash\{j\}}\left(\left(x_{i}-\bar{x}_{i}+1\right) \otimes_{L} \tilde{x}_{j}^{(i)}\right) \\
& =\left(\left(x_{j}-\bar{x}_{j}+1\right) \otimes_{L} \bar{x}_{j}\right) \oplus \bigoplus_{i \in N \backslash\{j\}}\left(\left(x_{i}-\bar{x}_{i}+1\right) \otimes_{L} \underline{x}_{j}\right) .
\end{aligned}
$$

In particular, $\left(x_{j}-\bar{x}_{j}+1\right) \otimes_{L} \bar{x}_{j}=\left(x_{j}-\bar{x}_{j}+1\right)+\bar{x}_{j}-1=x_{j}$, since $x_{j} \geq 0$. On the other hand, for $i \neq j$ we have $\left(x_{i}-\bar{x}_{i}+1\right) \otimes_{L} \underline{x}_{j}=\left(x_{i}-\bar{x}_{i}+1\right)+\underline{x}_{j}-1 \leq x_{j}$, because $x_{i}-\bar{x}_{i} \leq 0$.

For the converse implication, assume that $\underline{x}_{i}-\bar{x}_{i}+1 \leq \beta_{i} \leq 1$ for every $i \in N$ and $x=\bigoplus_{i \in N} \beta_{i} \otimes_{L} \tilde{x}^{(i)}$. For every $j \in N$

$$
\begin{gathered}
x_{j}=\left(\oplus_{i \in N} \beta_{i} \otimes_{L} \tilde{x}^{(i)}\right)_{j} \leq\left(\oplus_{i \in N} 1 \otimes_{L} \tilde{x}^{(i)}\right)_{j} \\
=\bigoplus_{i \in N} \tilde{x}_{j}^{(i)}=\bar{x}_{j} \oplus \bigoplus_{i \in N \backslash\{j\}} \underline{x}_{j}=\bar{x}_{j}, \\
x_{j}=\left(\bigoplus_{i \in N} \beta_{i} \otimes_{L} \tilde{x}^{(i)}\right)_{j} \geq \bigoplus_{i \in N}\left(\left(\underline{x}_{i}-\bar{x}_{i}+1\right) \otimes_{L} \tilde{x}^{(i)}\right)_{j} \\
=\left(\left(\underline{x}_{j}-\bar{x}_{j}+1\right)+\bar{x}_{j}-1\right) \oplus \bigoplus_{i \in N \backslash\{j\}}\left(\left(\underline{x}_{i}-\bar{x}_{i}+1\right)+\underline{x}_{j}-1\right) \\
\geq \underline{x}_{j} \oplus \bigoplus_{i \in N \backslash\{j\}}\left(\underline{x}_{i}-\bar{x}_{i}+\underline{x}_{j}\right)=\underline{x}_{j} .
\end{gathered}
$$

We have shown that $\underline{x} \leq x \leq \bar{x}$. That is, $x \in[\underline{x}, \bar{x}]$. The proof of (ii) is analogous.

Theorem 1. The interval vector $\mathbf{X}=[\underline{x}, \bar{x}]$ is a strong max- - uk eigenvector of the interval matrix $\mathbf{A}=[\underline{A}, \bar{A}]$ if and only if there exists $\lambda \in \mathcal{I}, \lambda>0$, such that for every $i \in N$.

$$
\begin{aligned}
& \underline{A} \otimes_{L} \tilde{x}^{(i)}=\lambda \otimes_{L} \tilde{x}^{(i)}, \\
& \bar{A} \otimes_{L} \tilde{x}^{(i)}=\lambda \otimes_{L} \tilde{x}^{(i)} .
\end{aligned}
$$


Proof. Assume that $\lambda \in \mathcal{I}$ fulfills conditions (6) and (7), and that $x \in \mathbf{X}$ is given. Then $x$ is a max-Łuk linear combination $x=\bigoplus_{i \in N} \beta_{i} \otimes_{L} \tilde{x}^{(i)}$ for some coefficients $\beta_{i} \in \mathcal{I}, i \in N$ with $\underline{x}_{i}-\bar{x}_{i}+1 \leq \beta_{i} \leq 1$, according to Lemma $1(\mathrm{i})$. In view of (6) we get

$$
\begin{aligned}
\underline{A} \otimes_{L} x & =\underline{A} \otimes_{L}\left(\bigoplus_{i \in N} \beta_{i} \otimes_{L} \tilde{x}^{(i)}\right)=\bigoplus_{i \in N}\left(\underline{A} \otimes_{L} \beta_{i} \otimes_{L} \tilde{x}^{(i)}\right) \\
& =\bigoplus_{i \in N} \beta_{i} \otimes_{L}\left(\underline{A} \otimes_{L} \tilde{x}^{(i)}\right)=\bigoplus_{i \in N} \beta_{i} \otimes_{L}\left(\lambda \otimes_{L} \tilde{x}^{(i)}\right) \\
& =\lambda \otimes_{L} \bigoplus_{i \in N}\left(\beta_{i} \otimes_{L} \tilde{x}^{(i)}\right)=\lambda \otimes_{L} x .
\end{aligned}
$$

Using (7) we analogously get

$$
\underline{A} \otimes_{L} x=\bar{A} \otimes_{L}\left(\bigoplus_{i \in N} \beta_{i} \otimes_{L} \tilde{x}^{(i)}\right)=\lambda \otimes_{L} \bigoplus_{i \in N}\left(\beta_{i} \otimes_{L} \tilde{x}^{(i)}\right)=\lambda \otimes_{L} x .
$$

From (8) and (9) it easily follows that

$$
\begin{gathered}
\lambda \otimes_{L} x=\underline{A} \otimes_{L} x \leq A \otimes_{L} x \leq \bar{A} \otimes_{L} x=\lambda \otimes_{L} x, \\
A \otimes_{L} x=\lambda \otimes_{L} x,
\end{gathered}
$$

for every $A \in \mathbf{A}$. That is, $\mathbf{X}$ is a strong max-Łuk eigenvector of $\mathbf{A}$. The converse implication is trivial.

Example 2. (Numerical illustration: Strong max-Luk eigenvector)

Assume lower and upper bounds for $A \in[\underline{A}, \bar{A}]$ and for $x \in[\underline{x}, \bar{x}]$

$$
\begin{gathered}
\underline{A}=\left(\begin{array}{ccccc}
0.6 & 0.2 & 0.1 & 0.1 & 0.5 \\
0.2 & 0.2 & 0.1 & 0 & 0.4 \\
0.1 & 0.3 & 0.2 & 0.4 & 0.2 \\
0.3 & 0.5 & 0.4 & 0.6 & 0.2 \\
0 & 0.1 & 0.3 & 0 & 0.3
\end{array}\right), \quad \bar{A}=\left(\begin{array}{ccccc}
0.6 & 0.6 & 0.3 & 0.1 & 0.6 \\
0.2 & 0.6 & 0.3 & 0.1 & 0.6 \\
0.5 & 0.9 & 0.6 & 0.4 & 0.9 \\
0.7 & 1 & 0.8 & 0.6 & 0.9 \\
0.2 & 0.6 & 0.3 & 0.1 & 0.6
\end{array}\right) \\
\underline{x}=\left(\begin{array}{c}
0 \\
0 \\
0.7 \\
0.9 \\
0
\end{array}\right), \quad \bar{x}=\left(\begin{array}{c}
0.8 \\
0.4 \\
0.7 \\
0.9 \\
0.4
\end{array}\right) .
\end{gathered}
$$

Then

$$
\tilde{x}^{(1)}=\left(\begin{array}{c}
0.8 \\
0 \\
0.7 \\
0.9 \\
0
\end{array}\right), \quad \tilde{x}^{(2)}=\left(\begin{array}{c}
0 \\
0.4 \\
0.7 \\
0.9 \\
0
\end{array}\right), \quad \tilde{x}^{(3)}=\left(\begin{array}{c}
0 \\
0 \\
0.7 \\
0.9 \\
0
\end{array}\right), \quad \tilde{x}^{(4)}=\left(\begin{array}{c}
0 \\
0 \\
0.7 \\
0.9 \\
0
\end{array}\right), \quad \tilde{x}^{(5)}=\left(\begin{array}{c}
0 \\
0 \\
0.7 \\
0.9 \\
0.4
\end{array}\right) \text {. }
$$

The following equations hold for $\lambda=0.6$ 


$$
\begin{aligned}
& \underline{A} \otimes_{L} \tilde{x}^{(1)}=\left(\begin{array}{c}
0.4 \\
0 \\
0.3 \\
0.5 \\
0
\end{array}\right)=\bar{A} \otimes_{L} \tilde{x}^{(1)}=0.6 \otimes_{L} \tilde{x}^{(1)}, \\
& \underline{A} \otimes_{L} \tilde{x}^{(2)}=\left(\begin{array}{c}
0 \\
0 \\
0.3 \\
0.5 \\
0
\end{array}\right)=\bar{A} \otimes_{L} \tilde{x}^{(2)}=0.6 \otimes_{L} \tilde{x}^{(2)}, \\
& \underline{A} \otimes_{L} \tilde{x}^{(3)}=\left(\begin{array}{c}
0 \\
0 \\
0.3 \\
0.5 \\
0
\end{array}\right)=\bar{A} \otimes_{L} \tilde{x}^{(3)}=0.6 \otimes_{L} \tilde{x}^{(3)}, \\
& \underline{A} \otimes_{L} \tilde{x}^{(4)}=\left(\begin{array}{c}
0 \\
0 \\
0.3 \\
0.5 \\
0
\end{array}\right)=\bar{A} \otimes_{L} \tilde{x}^{(4)}=0.6 \otimes_{L} \tilde{x}^{(4)}, \\
& \underline{A} \otimes_{L} \tilde{x}^{(5)}=\left(\begin{array}{c}
0 \\
0 \\
0.3 \\
0.5 \\
0
\end{array}\right)=\bar{A} \otimes_{L} \tilde{x}^{(5)}=0.6 \otimes_{L} \tilde{x}^{(5)} .
\end{aligned}
$$

Hence, $\mathbf{X}=[\underline{x}, \bar{x}]$ is a strong max- $\mathrm{tuk}$ eigenvector of $A$ with the eigenvalue $\lambda=0.6$.

Theorem 1 leads to the following recognition problem: given $\mathbf{A}$ and $\mathbf{X}$, recognize whether there is, or is no value $\lambda \in \mathcal{I} \backslash\{0\}$ such that (6) and (7) hold for every $k \in N$. If the answer is positive, then find all (or at least one) such values.

If $i, k \in N$, then we write, for brevity,

$$
\begin{aligned}
& z_{i k}=\left(\underline{A} \otimes_{L} \tilde{x}^{(i)}\right)_{k}, \\
& z_{i k}^{\prime}=\left(\bar{A} \otimes_{L} \tilde{x}^{(i)}\right)_{k} .
\end{aligned}
$$

Furthermore, we write

$$
\begin{aligned}
Z^{0} & =\left\{(i, k) \in N \times N ; z_{i k}=0\right\} \\
Z^{>} & =\left\{(i, k) \in N \times N ; z_{i k}>0\right\} \\
I & =\left(0, \min _{(i, k) \in N \times N}\left(1-\tilde{x}_{k}^{(i)}\right)\right\}
\end{aligned}
$$

Theorem 2. The interval vector $\mathbf{X}=[\underline{x}, \bar{x}]$ is a strong max-Euk eigenvector of the interval matrix $\mathbf{A}=[\underline{A}, \bar{A}]$ if and only if

(i) $\quad(\forall(i, k) \in N \times N) \quad z_{i k}=z_{i k^{\prime}}^{\prime}$

(ii) $\left(\forall(i, k) \in Z^{0}\right) \quad \tilde{x}_{k}^{(i)}<1$, 
(iii) $\quad\left(\forall(i, k),(j, l) \in Z^{>}\right) \quad \tilde{x}_{k}^{(i)}-z_{i k}=\tilde{x}_{l}^{(j)}-z_{j l}<1$,

(iv) $\quad\left(\forall(i, k) \in Z^{>},(j, l) \in Z^{0}\right) \quad \tilde{x}_{l}^{(j)} \leq \tilde{x}_{k}^{(i)}-z_{i k}$.

Proof. Assume that $\mathbf{X}$ is a strong interval eigenvector of $\mathbf{A}$. That is, there exists $\lambda \in \mathcal{I}, \lambda>0$ fulfilling conditions (6) and (7). The statement (i) then follows immediately. For $(i, k) \in Z^{0}$ we have $z_{i k}=0$, which gives $\lambda \otimes_{L} \tilde{x}_{k}^{(i)}=0$, in view of (6). Then, by definition of $\otimes_{L}$, we have $\lambda+\tilde{x}_{k}^{(i)}-1 \leq 0$, which implies $\lambda \leq 1-\tilde{x}_{k}^{(i)}$. Now, statement (ii) easily follows, in view of the assumption that $0<\lambda$.

For $(i, k),(j, l) \in Z^{>}$, we have $z_{i k}>0$, which gives $\lambda \otimes_{L} \tilde{x}_{k}^{(i)}>0$, in view of (6). Consequently, $z_{i k}=\lambda+\tilde{x}_{k}^{(i)}-1$. That is, $\lambda=-\tilde{x}_{k}^{(i)}+z_{i k}+1$. Similarly, $\lambda=-\tilde{x}_{l}^{(j)}+z_{j l}+1$. In view of the assumption that $\lambda>0$, we get (iii) by a simple computation. Finally, assume $(i, k) \in Z^{0},(j, l) \in Z^{>}$. By the same arguments as above, we get $\lambda=-\tilde{x}_{k}^{(i)}+z_{i k}+1 \leq 1-\tilde{x}_{l}^{(j)}$. Then (iv) follows directly.

For the converse implication, assume that statements (i)-(iv) hold. We shall show that then a $\lambda>0$ can be found such that (6) and (7) are satisfied. We distinguish two cases.

Case 1. $Z^{>}=\varnothing$. Then $Z^{0}=N \times N$, and by (ii) we have $0<1-\tilde{x}_{k}^{(i)}$ for every $(i, k) \in N \times N$. That is, the interval $I$ is non-empty. Choose an arbitrary $\lambda \in I$. Then, for every $(i, k) \in N \times N$, we have $\lambda \leq 1-\tilde{x}_{k}^{(i)}$ which gives $\lambda+\tilde{x}_{k}^{(i)}-1 \leq 0$. That is, $\lambda \otimes_{L} \tilde{x}_{k}^{(i)}=0=z_{i k}$. As $(i, k)$ is arbitrary, (6) has been demonstrated. Then, (7) follows by statement (i) .

Case 2. $\quad Z^{>} \neq \varnothing$. Let $(i, k) \in Z^{>}$be fixed. By (iii) we have $\tilde{x}_{k}^{(i)}-z_{i k}<1$, which gives $0<$ $z_{i k}+1-\tilde{x}_{k}^{(i)}$. Choosing $\lambda=z_{i k}+1-\tilde{x}_{k}^{(i)}$, we get $\lambda>0$ and $z_{i k}=\lambda+\tilde{x}_{k}^{(i)}-1$. Then, the assumption that $(i, k) \in Z^{>}$implies $z_{i k}>0$ and $\lambda+\tilde{x}_{k}^{(i)}-1>0$. That is, $\lambda+\tilde{x}_{k}^{(i)}-1=\lambda \otimes_{L} \tilde{x}_{k}^{(i)}$, which implies $z_{i k}=\lambda \otimes_{L} \tilde{x}_{k}^{(i)}$.

Consider an arbitrary $(j, l) \in Z^{>}$. We have $\lambda=z_{i k}+1-\tilde{x}_{k}^{(i)}=z_{j l}+1-\tilde{x}_{l}^{(j)}$, in view of (iii). That is, $z_{j l}=\lambda \otimes_{L} \tilde{x}_{l}^{(j)}$, similarly as above. On the other hand, for every $(j, l) \in Z^{0}$ we have $\tilde{x}_{l}^{(j)} \leq \tilde{x}_{k}^{(i)}-z_{i k}$, in view of (iv). Consequently, we get $z_{i k}+1-\tilde{x}_{k}^{(i)} \leq 1-\tilde{x}_{l}^{(j)}$. That is, $\lambda \leq 1-\tilde{x}_{l}^{(j)}$, which gives $\lambda+\tilde{x}_{l}^{(j)}-1 \leq 0$. This implies $\lambda \otimes_{L} \tilde{x}_{l}^{(j)}=0$, i.e., $z_{j l}=\lambda \otimes_{L} \tilde{x}_{l}^{(j)}$. As $(j, l) \in Z^{>} \cup Z^{0}=N \times N$ was arbitrary, we have shown that (6) is satisfied. By (i), (7) holds as well.

Remark 3. The proof of Theorem 2 contains a description of the set $S(\mathbf{A}, \mathbf{X})=\{\lambda>0 ;(\forall A \in$ A) $\left.(\forall x \in \mathbf{X}) A \otimes_{L} x=\lambda \otimes_{L} x\right\}$. Namely

(i) if some of statements (i)-(iv) in Theorem 2 are not satisfied, then $S(\mathbf{A}, \mathbf{X})=\varnothing$,

(ii) $\quad$ if $(i, k) \in Z^{>}$, then $S(\mathbf{A}, \mathbf{X})=\{\lambda\}=\left\{z_{i k}+1-\tilde{x}_{k}^{(i)}\right\}$,

(iii) if $Z^{>}=\varnothing$, then $S(\mathbf{A}, \mathbf{X})=\left(0, \min _{i, k \in N}\left(1-\tilde{x}_{k}^{(i)}\right)\right\rangle$.

Theorem 3. The recognition problem of whether a given interval vector $\mathbf{X}$ is a strong max- $\mathrm{tuk}$ eigenvector of the interval matrix $\mathbf{A}$ is solvable in $O\left(n^{3}\right)$ time.

Proof. According to Theorem 2, the problem can be solved by verifying conditions (i)-(iv). Each of them can be verified in $O\left(n^{3}\right)$ time. Therefore, the computational complexity is $O\left(n^{3}\right)$.

\section{Strongly Tolerable Interval Eigenvectors in a Max-Łuk Algebra}

Theorem 4. The interval vector $\mathbf{X}=[\underline{\mathbf{x}}, \overline{\mathbf{x}}]$ is a strongly tolerable max-Euk eigenvector of the interval matrix $\mathbf{A}=[\underline{A}, \bar{A}]$ if and only if there exist an $A \in \mathbf{A}$ and $\lambda \in \mathcal{I}$ such that

$$
A \otimes_{L} \tilde{x}^{(k)}=\lambda \otimes_{L} \tilde{x}^{(k)} \quad \text { for every } k \in N .
$$


Proof. Let us assume that $A \in \mathbf{A}$ and $\lambda \in \mathcal{I}$ fulfill condition (15). If $x \in \mathcal{I}(n)$ is an arbitrary vector in $\mathbf{X}$, then $x$ is a max- $\succeq u k$ linear combination $x=\bigoplus_{k \in N} \beta_{k} \otimes_{L} \tilde{x}^{(k)}$ for some coefficients $\beta_{k} \in \mathcal{I}, k \in N$ with $\underline{x}_{k}-\bar{x}_{k}+1 \leq \beta_{i} \leq 1$. According to Lemma 1 (i),

$$
\begin{aligned}
A \otimes_{L} x & =A \otimes_{L}\left(\oplus_{k \in N} \beta_{k} \otimes_{L} \tilde{x}^{(k)}\right)=\bigoplus_{k \in N}\left(A \otimes_{L} \beta_{k} \otimes_{L} \tilde{x}^{(k)}\right) \\
& =\bigoplus_{k \in N} \beta_{k} \otimes_{L}\left(A \otimes_{L} \tilde{x}^{(k)}\right)=\bigoplus_{k \in N} \beta_{k} \otimes_{L}\left(\lambda \otimes_{L} \tilde{x}^{(k)}\right) \\
& =\lambda \otimes_{L} \bigoplus_{k \in N}\left(\beta_{k} \otimes_{L} \tilde{x}^{(k)}\right)=\lambda \otimes_{L} x .
\end{aligned}
$$

By (3), $\mathbf{X}$ is a strongly tolerable eigenvector of $\mathbf{A}$. The converse implication follows immediately.

Remark 4. The property (15) can be briefly expressed in words: $A$ is a $\lambda$-certificate for the strong tolerance max-Łuk problem $(\mathbf{A}, \mathbf{X})$.

Example 3. (Numerical illustration: Strongly tolerable max-Luk eigenvector)

Assume the lower and upper bounds for $A \in[\underline{A}, \bar{A}]$ and for $x \in[\underline{x}, \bar{x}]$ are

$$
\begin{array}{cc}
\underline{A}=\left(\begin{array}{ccccc}
0.2 & 0.2 & 0.2 & 0.3 & 0 \\
0.1 & 0.3 & 0.2 & 0.3 & 0 \\
0 & 0.1 & 0.2 & 0.3 & 0.1 \\
0.1 & 0.2 & 0.1 & 0.2 & 0.2 \\
0.1 & 0.1 & 0.2 & 0.1 & 0.4
\end{array}\right), \quad \bar{A}=\left(\begin{array}{lllll}
0.8 & 0.8 & 0.9 & 0.7 & 0.5 \\
0.5 & 0.8 & 0.9 & 0.8 & 0.5 \\
0.5 & 0.7 & 0.5 & 0.5 & 0.8 \\
0.5 & 0.5 & 0.5 & 0.8 & 0.8 \\
0.5 & 0.2 & 0.9 & 0.9 & 0.8
\end{array}\right) \\
\underline{x}=\left(\begin{array}{c}
0.6 \\
0 \\
0.6 \\
0 \\
0.7
\end{array}\right), \quad \bar{x}=\left(\begin{array}{c}
0.8 \\
0.7 \\
0.6 \\
0.4 \\
0.7
\end{array}\right) .
\end{array}
$$

Then

$$
\tilde{x}^{(1)}=\left(\begin{array}{c}
0.8 \\
0 \\
0.6 \\
0 \\
0.7
\end{array}\right), \quad \tilde{x}^{(2)}=\left(\begin{array}{c}
0.6 \\
0.7 \\
0.6 \\
0 \\
0.7
\end{array}\right), \quad \tilde{x}^{(3)}=\left(\begin{array}{c}
0.6 \\
0 \\
0.6 \\
0 \\
0.7
\end{array}\right), \quad \tilde{x}^{(4)}=\left(\begin{array}{c}
0.6 \\
0 \\
0.6 \\
0.4 \\
0.7
\end{array}\right), \quad \tilde{x}^{(5)}=\left(\begin{array}{c}
0.6 \\
0 \\
0.6 \\
0 \\
0.7
\end{array}\right) .
$$

For $\lambda=0.7$ and for given $A_{1} \in \mathbf{A}$

$$
A_{1}=\left(\begin{array}{lllll}
0.7 & 0.6 & 0.7 & 0.6 & 0.1 \\
0.2 & 0.7 & 0.4 & 0.6 & 0.1 \\
0.2 & 0.2 & 0.3 & 0.4 & 0.6 \\
0.2 & 0.3 & 0.4 & 0.7 & 0.3 \\
0.2 & 0.1 & 0.8 & 0.8 & 0.4
\end{array}\right)
$$

the following equations hold

$$
A_{1} \otimes_{L} \tilde{x}^{(1)}=\left(\begin{array}{ccccc}
0.7 & 0.6 & 0.7 & 0.6 & 0.1 \\
0.2 & 0.7 & 0.4 & 0.6 & 0.1 \\
0.2 & 0.2 & 0.3 & 0.4 & 0.6 \\
0.2 & 0.3 & 0.4 & 0.7 & 0.3 \\
0.2 & 0.1 & 0.8 & 0.8 & 0.4
\end{array}\right) \otimes\left(\begin{array}{c}
0.8 \\
0 \\
0.6 \\
0 \\
0.7
\end{array}\right)=\left(\begin{array}{c}
0.5 \\
0 \\
0.3 \\
0 \\
0.4
\end{array}\right)=0.7 \otimes_{L} \tilde{x}^{(1)}
$$




$$
\begin{aligned}
& A_{1} \otimes_{L} \tilde{x}^{(2)}=\left(\begin{array}{ccccc}
0.7 & 0.6 & 0.7 & 0.6 & 0.1 \\
0.2 & 0.7 & 0.4 & 0.6 & 0.1 \\
0.2 & 0.2 & 0.3 & 0.4 & 0.6 \\
0.2 & 0.3 & 0.4 & 0.7 & 0.3 \\
0.2 & 0.1 & 0.8 & 0.8 & 0.4
\end{array}\right) \otimes\left(\begin{array}{c}
0.6 \\
0.7 \\
0.6 \\
0 \\
0.7
\end{array}\right)=\left(\begin{array}{c}
0.3 \\
0.4 \\
0.3 \\
0 \\
0.4
\end{array}\right)=0.7 \otimes_{L} \tilde{x}^{(2)} \\
& A_{1} \otimes_{L} \tilde{x}^{(3)}=\left(\begin{array}{ccccc}
0.7 & 0.6 & 0.7 & 0.6 & 0.1 \\
0.2 & 0.7 & 0.4 & 0.6 & 0.1 \\
0.2 & 0.2 & 0.3 & 0.4 & 0.6 \\
0.2 & 0.3 & 0.4 & 0.7 & 0.3 \\
0.2 & 0.1 & 0.8 & 0.8 & 0.4
\end{array}\right) \otimes\left(\begin{array}{c}
0.6 \\
0 \\
0.6 \\
0 \\
0.7
\end{array}\right)=\left(\begin{array}{c}
0.3 \\
0 \\
0.3 \\
0 \\
0.4
\end{array}\right)=0.7 \otimes_{L} \tilde{x}^{(3)}, \\
& A_{1} \otimes_{L} \tilde{x}^{(4)}=\left(\begin{array}{ccccc}
0.7 & 0.6 & 0.7 & 0.6 & 0.1 \\
0.2 & 0.7 & 0.4 & 0.6 & 0.1 \\
0.2 & 0.2 & 0.3 & 0.4 & 0.6 \\
0.2 & 0.3 & 0.4 & 0.7 & 0.3 \\
0.2 & 0.1 & 0.8 & 0.8 & 0.4
\end{array}\right) \otimes\left(\begin{array}{c}
0.6 \\
0 \\
0.6 \\
0.4 \\
0.7
\end{array}\right)=\left(\begin{array}{c}
0.3 \\
0 \\
0.3 \\
0.1 \\
0.4
\end{array}\right)=0.7 \otimes_{L} \tilde{x}^{(4)}, \\
& A_{1} \otimes_{L} \tilde{x}^{(5)}=\left(\begin{array}{ccccc}
0.7 & 0.6 & 0.7 & 0.6 & 0.1 \\
0.2 & 0.7 & 0.4 & 0.6 & 0.1 \\
0.2 & 0.2 & 0.3 & 0.4 & 0.6 \\
0.2 & 0.3 & 0.4 & 0.7 & 0.3 \\
0.2 & 0.1 & 0.8 & 0.8 & 0.4
\end{array}\right) \otimes\left(\begin{array}{c}
0.6 \\
0 \\
0.6 \\
0 \\
0.7
\end{array}\right)=\left(\begin{array}{c}
0.3 \\
0 \\
0.3 \\
0 \\
0.4
\end{array}\right)=0.7 \otimes_{L} \tilde{x}^{(5)},
\end{aligned}
$$

Hence, $\mathbf{X}=[\underline{x}, \bar{x}]$ is a strongly tolerable max- - uk eigenvector of $\mathbf{A}$ with the eigenvalue $\lambda=0.7$, and $A_{1}$ is the $\lambda$-certificate for the strong tolerance max-Łuk problem $(\mathbf{A}, \mathbf{X})$.

Remark 5. In general, not every matrix $A \in \mathbf{A}$ is a $\lambda$-certificate for $(\mathbf{A}, \mathbf{X})$, for some $\lambda$. Take, e.g.,

$$
A_{2}=\left(\begin{array}{lllll}
0.8 & 0.8 & 0.9 & 0.7 & 0.5 \\
0.5 & 0.8 & 0.9 & 0.8 & 0.5 \\
0.5 & 0.7 & 0.5 & 0.5 & 0.8 \\
0.5 & 0.5 & 0.5 & 0.8 & 0.8 \\
0.5 & 0.2 & 0.9 & 0.9 & 0.8
\end{array}\right)
$$

and $\tilde{x}^{(1)}, \tilde{x}^{(2)}, \tilde{x}^{(3)}, \tilde{x}^{(4)}, \tilde{x}^{(5)}$ from Example 3. Then

$$
A_{2} \otimes_{L} \tilde{x}^{(1)}=A_{2} \otimes\left(\begin{array}{c}
0.8 \\
0 \\
0.6 \\
0 \\
0.7
\end{array}\right)=\left(\begin{array}{c}
0 \\
0 \\
0 \\
0 \\
0.1
\end{array}\right) \neq \lambda \otimes_{L} \tilde{x}^{(1)} .
$$

It is easy to see that the equality in the last position cannot hold for any $\lambda \in \mathcal{I}$. That is, $A_{2}$ is not a $\lambda$-certificate in Example 3 with any $\lambda \in \mathcal{I}$.

In Example 3, the certificate $A_{1}$ was given. Now the question arises of how to find a certificate (or to show that no certificate exists) for a given instance $(\mathbf{A}, \mathbf{X})$. In other words, how do we recognize whether or not $\mathbf{X}$ is a strongly tolerable interval eigenvector of $\mathbf{A}$ ?

A method for solving the strong tolerance interval eigenproblem in a max- $€$ uk algebra for instances with a natural additional condition is described in the rest of this section. We start with a simple lemma. 
Lemma 2. Assume $u, v, w \in \mathcal{I}$.

(i) If $u+v+w>2$, then $\left(u \otimes_{L} v\right) \otimes_{L} w=u+v+w-2>0$,

(ii) If $u+v+w \leq 2$, then $\left(u \otimes_{L} v\right) \otimes_{L} w=0$.

Proof. Let $u+v+w>2$. Then $u+v>2-w \geq 2-1=1$, since $0 \leq w \leq 1$. Hence, $u \otimes_{L} v=$ $u+v-1>0$, and $\left(u \otimes_{L} v\right) \otimes_{L} w=(u+v-1)+w-1=u+v+w-2>0$.

On the other hand, if $u+v+w \leq 2$, then $(u+v-1)+(w-1) \leq 0$. We consider two subcases.

Subcase 1. Suppose $u+v-1>0$. Then $u \otimes_{L} v=u+v-1$ and $u \otimes_{L} v+w-1 \leq 0$. Hence, $\left(u \otimes_{L} v\right) \otimes_{L} w=0$.

Subcase 2. Suppose $u+v-1 \leq 0$. Then $u \otimes_{L} v=0$ and $u \otimes_{L} v+w-1=0+w-1 \leq 0$, since $w \leq 1$. That is, $\left(u \otimes_{L} v\right) \otimes_{L} w=0$.

Remark 6. It is easy to see directly from the definition that the Eukasiewicz conjunction $\otimes_{L}$ is commutative. As a consequence of Lemma $2, \otimes_{L}$ is associative, as well.

Namely due to Lemma 2(i), we have, for any $u, v, w \in \mathcal{I}$ with $u+v+w>2$, that $\left(u \otimes_{L} v\right) \otimes_{L} w=$ $u+v+w-2$, and, by the commutative law, $u \otimes_{L}\left(v \otimes_{L} w\right)=\left(v \otimes_{L} w\right) \otimes_{L} u=v+w+u-2$. That is, $\left(u \otimes_{L} v\right) \otimes_{L} w=u \otimes_{L}\left(v \otimes_{L} w\right)$. Similar reasoning is used when $u+v+w \leq 2$.

To recognize the existence of a certificate $A \in \mathbf{A}$ satisfying the conditions (15) from Theorem 4, the unknown $A$ will be written as a max-Łuk linear combination of generators $\tilde{A}^{(i j)}$ as in Lemma 1 (ii).

The coefficients in the linear combination will be found as the solution to a system of max-Łuk linear equations with parameter $\lambda$, and the variables $\alpha_{(i j)}$ in the bounds $\underline{a}_{i j}=\underline{\alpha}_{(i j)} \leq \alpha_{(i j)} \leq \bar{\alpha}_{(i j)}=\bar{a}_{i j}$. The form of the system will require that every solution of the system, for some parameter value $\lambda$, gives coefficients for such a max-Łuk linear combination of generators which is a $\lambda$-certificate matrix for the given instance. Then the recognition of strong tolerability is equivalent to the recognition of whether there is a value $\lambda$ for which the system is solvable. On the other hand, if the system is unsolvable for every $\lambda \in \mathcal{I}$, then no certificate exists for the given instance.

Formally, we consider the bounded max-Łuk linear system

$$
\begin{aligned}
\tilde{C} \otimes_{L} \alpha & =\lambda \otimes_{L} \tilde{b} \\
\underline{\alpha} & \leq \alpha \leq \bar{\alpha}
\end{aligned}
$$

with parameter $\lambda \in \mathcal{I}$, where the columns $\tilde{C}^{(i j)}$ of $\tilde{C} \in \mathcal{I}\left(n^{2}, n^{2}\right)$ are constructed blockwise from $\tilde{A}^{(i j)} \otimes \tilde{x}^{(k)}, k \in N$. The right-hand side vector $\tilde{b} \in \mathcal{I}\left(n^{2}\right)$ is constructed blockwise from the generators $\tilde{x}^{(k)}$ for $k \in N$, and the bounds $\underline{\alpha}, \bar{\alpha} \in \mathcal{I}\left(n^{2}\right)$ for the variable vector $\alpha \in \mathcal{I}\left(n^{2}\right)$ are constructed from the columns of $\underline{A}, \bar{A}$, according to Lemma 1 (ii). That is, we have

$$
\begin{gathered}
\tilde{C}^{(i j)}=\left(\begin{array}{c}
\tilde{A}^{(i j)} \otimes_{L} \tilde{x}^{(1)} \\
\tilde{A}^{(i j)} \otimes_{L} \tilde{x}^{(2)} \\
\vdots \\
\tilde{A}^{(i j)} \otimes_{L} \tilde{x}^{(n)}
\end{array}\right), \quad \tilde{b}=\left(\begin{array}{c}
\tilde{x}^{(1)} \\
\tilde{x}^{(2)} \\
\vdots \\
\tilde{x}^{(n)}
\end{array}\right), \\
\underline{\alpha}_{(i j)}=\underline{a}_{i j}-\bar{a}_{i j}+1 \leq \alpha_{(i j)} \leq 1=\bar{\alpha}_{(i j)} .
\end{gathered}
$$


Theorem 5. The interval vector $\mathbf{X}=[\underline{x}, \bar{x}]$ is a strongly tolerable eigenvector of the interval matrix $\mathbf{A}=[\underline{A}, \bar{A}]$ if and only if there is a $\lambda \in \mathcal{I}$ such that the linear system (17) and (18), has a solution $\alpha \in \mathcal{I}\left(n^{2}\right)$. In the positive case, the max- $\succeq$ uk linear combination

$$
A=\bigoplus_{(i j) \in N \times N} \alpha_{(i j)} \otimes_{L} \tilde{A}^{(i j)}
$$

is a $\lambda$-certificate for the given instance.

Proof. Assume that there exists a $\lambda \in \mathcal{I}$ such that $\alpha$ satisfies (17), (18) with (19), (20). Then, $A \in \mathcal{I}(n, n)$ as defined in (21) belongs to $[\underline{A}, \bar{A}]$, in view of Lemma 1(ii). Moreover, we have the following block equations, for every $k \in N$

$$
\begin{aligned}
\bigoplus_{i, j \in N}\left(\tilde{A}^{(i j)} \otimes_{L} \tilde{x}^{(k)}\right) \otimes_{L} \alpha_{(i j)} & =\lambda \otimes_{L} \tilde{x}^{(k)}, \\
\left(\bigoplus_{i, j \in N} \alpha_{(i j)} \otimes_{L} \tilde{A}^{(i j)}\right) \otimes_{L} \tilde{x}^{(k)} & =\lambda \otimes_{L} \tilde{x}^{(k)}, \\
A \otimes_{L} \tilde{x}^{(k)} & =\lambda \otimes_{L} \tilde{x}^{(k)} .
\end{aligned}
$$

We will prove that the block Equations (22) and (23) are equivalent. In particular, we show that the left-hand sides of (22) and (23) in every row $h$ and in every block row $k$ are equal.

Assume $k, h \in N$ are fixed. Then

$$
\begin{aligned}
& \left(\bigoplus_{i, j \in N}\left(\tilde{A}^{(i j)} \otimes_{L} \tilde{x}^{(k)}\right) \otimes_{L} \alpha_{(i j)}\right)_{h}=\bigoplus_{i, j \in N}\left(\tilde{A}^{(i j)} \otimes_{L} \tilde{x}^{(k)}\right)_{h} \otimes_{L} \alpha_{(i j)} \\
& =\bigoplus_{i, j \in N}\left(\bigoplus_{g \in N} \tilde{A}_{h g}^{(i j)} \otimes_{L} \tilde{x}_{g}^{(k)}\right) \otimes_{L} \alpha_{(i j)}=\bigoplus_{i, j \in N}\left(\bigoplus_{g \in N}\left(\tilde{A}_{h g}^{(i j)} \otimes_{L} \tilde{x}_{g}^{(k)}\right) \otimes_{L} \alpha_{(i j)}\right) \\
& =\bigoplus_{i, j \in N}\left(\bigoplus_{g \in N} \alpha_{(i j)} \otimes_{L}\left(\tilde{A}_{h g}^{(i j)} \otimes_{L} \tilde{x}_{g}^{(k)}\right)\right)=\bigoplus_{i, j \in N}\left(\bigoplus_{g \in N}\left(\alpha_{(i j)} \otimes_{L} \tilde{A}_{h g}^{(i j)}\right) \otimes_{L} \tilde{x}_{g}^{(k)}\right) \\
& =\bigoplus_{i, j \in N}\left(\alpha_{(i j)} \otimes_{L} \tilde{A}^{(i j)}\right)_{h} \otimes_{L} \tilde{x}^{(k)}=\left(\bigoplus_{i, j \in N}\left(\alpha_{(i j)} \otimes_{L} \tilde{A}^{(i j)}\right) \otimes_{L} \tilde{x}^{(k)}\right)_{h}
\end{aligned}
$$

Please note that the associative law has been used in (27). That is,

$$
\alpha_{(i j)} \otimes_{L}\left(\tilde{A}_{h g}^{(i j)} \otimes_{L} \tilde{x}_{g}^{(k)}\right)=\left(\alpha_{(i j)} \otimes_{L} \tilde{A}_{h g}^{(i j)}\right) \otimes_{L} \tilde{x}_{g}^{(k)},
$$

according to Remark 6. The remaining equalities (25), (26) and (28) are consequences of standard arithmetic rules in max-Łuk algebras.

Now, in view of the fact that (22) means that $\alpha \in \mathcal{I}\left(n^{2}\right)$ is a solution of (19), while (23) says that (21) satisfies (15), we obtain, due to Theorem 4 that $\mathbf{X}$ is a strongly tolerable eigenvector of $\mathbf{A}$. The converse implication follows from the converse implication in Theorem 4.

Theorem 5 reduces the recognition problem of whether $\mathbf{X}$ is a strongly tolerable eigenvector of A to the solvability problem of the bounded parametric system (17), (18) with dimension $n^{2} \times n^{2}$ for some $\lambda \in \mathcal{I}$. The latter problem is a particular case of the bounded parametric solvability problem with general dimension $m \times n$. The recognition algorithm can be briefly described by the following steps (for details and notation, see [28]): 
(i) permute the equations in the system so that the right-hand side will be decreasing, that is

$$
0 \leq 1-b_{1} \leq 1-b_{2} \leq \cdots \leq 1-b_{m} \leq 1
$$

(ii) recognize the solvability for some $\lambda$ with $1-b_{m}<\lambda \leq 1$, according to [28]/Theorem 3 (case a), by verifying $C \otimes_{L} y^{\star}\left(\lambda_{\max }^{m}\right)=\lambda_{\max }^{m} \otimes_{L} b$,

(iii) recognize the solvability for some $\lambda$ with $0 \leq \cdots \leq 1-b_{h}<\lambda \leq 1-b_{h+1} \leq \ldots 1$, according to [28]/Theorem 4 (case b), by verifying $C \otimes_{L} y^{\star}\left(\lambda_{\max }^{h}\right)=\lambda_{\max }^{h} \otimes_{L} b$. This step may be repeated, if necessary, with different indices $h \leq m$,

(iv) recognize the solvability for some $\lambda$ with $0 \leq \lambda \leq 1-b_{1}$, according to [28]/Theorem 5 (case c), by verifying $\underline{y}_{j} \leq \bigwedge_{i \in M}\left(1-c_{i j}\right)$, for every $j \in N$.

(v) the system is solvable if the answer is positive at least once in steps 2, 3 or 4 . Otherwise, the system is unsolvable for any value of $\lambda$.

Theorem 6. The recognition problem of whether a given interval vector $\mathbf{X}$ is a strongly tolerable eigenvector of a given interval matrix $\mathbf{A}$ in a max- $\leftarrow$ uk algebra is solvable in $O\left(n^{6}\right)$ time.

Proof. According to [28], the parametric solvability problem with dimension $m \times n$ has the computational complexity $O\left(m n^{2}\right)$. Therefore, the computational complexity of the strong tolerance problem with dimension $n^{2} \times n^{2}$ is $O\left(\left(n^{2}\right)^{3}\right)=O\left(n^{6}\right)$.

Example 4. (Numerical illustration: Computing a certificate)

Assume that the lower and upper bounds for $A \in[\underline{A}, \bar{A}]$ and $x \in[\underline{x}, \bar{x}]$ in the interval eigenproblem are

$$
\begin{array}{cc}
\underline{A}=\left(\begin{array}{lll}
0.9 & 0.7 & 0.6 \\
0.7 & 0.9 & 0.6 \\
0.8 & 0.8 & 0.9
\end{array}\right), & \bar{A}=\left(\begin{array}{ccc}
1 & 0.8 & 0.8 \\
0.7 & 0.9 & 0.8 \\
1 & 0.9 & 1
\end{array}\right) \\
\underline{x}=\left(\begin{array}{l}
0.7 \\
0.8 \\
0.9
\end{array}\right), \quad \bar{x}=\left(\begin{array}{l}
0.9 \\
0.8 \\
0.9
\end{array}\right) . &
\end{array}
$$

If we wish to recognize whether $\mathbf{X}$ is a strongly tolerable max- $\mathrm{t}$ uk eigenvector of $\mathbf{A}$, then, according to Theorem 4, we must recognize the existence of a $\lambda$-certificate for $(\mathbf{A}, \mathbf{X})$. In view of Theorem 5 , we must recognize the solvability of the max-Łuk linear system $\tilde{C} \otimes_{L} \alpha=\lambda \otimes_{L} \tilde{b}$ with bounds $\underline{\alpha} \leq \alpha \leq \bar{\alpha}$, for some $\lambda \in \mathcal{I}$. The vector (matrix) generators are

$$
\begin{array}{ll}
\tilde{x}^{(1)}=\left(\begin{array}{l}
0.9 \\
0.8 \\
0.9
\end{array}\right), \quad \tilde{x}^{(2)}=\left(\begin{array}{l}
0.7 \\
0.8 \\
0.9
\end{array}\right), \quad \tilde{x}^{(3)}=\left(\begin{array}{l}
0.7 \\
0.8 \\
0.9
\end{array}\right) . \\
\tilde{A}^{(11)}=\left(\begin{array}{ccc}
1 & 0.7 & 0.6 \\
0.7 & 0.9 & 0.6 \\
0.8 & 0.8 & 0.9
\end{array}\right), & \tilde{A}^{(12)}=\left(\begin{array}{lll}
0.9 & 0.8 & 0.6 \\
0.7 & 0.9 & 0.6 \\
0.8 & 0.8 & 0.9
\end{array}\right), \\
\tilde{A}^{(13)}=\left(\begin{array}{lll}
0.9 & 0.7 & 0.8 \\
0.7 & 0.9 & 0.6 \\
0.8 & 0.8 & 0.9
\end{array}\right), & \tilde{A}^{(21)}=\left(\begin{array}{lll}
0.9 & 0.7 & 0.6 \\
0.7 & 0.9 & 0.6 \\
0.8 & 0.8 & 0.9
\end{array}\right),
\end{array}
$$




$$
\begin{array}{ll}
\tilde{A}^{(22)}=\left(\begin{array}{ccc}
0.9 & 0.7 & 0.6 \\
0.7 & 0.9 & 0.6 \\
0.8 & 0.8 & 0.9
\end{array}\right), & \tilde{A}^{(23)}=\left(\begin{array}{lll}
0.9 & 0.7 & 0.6 \\
0.7 & 0.9 & 0.8 \\
0.8 & 0.8 & 0.9
\end{array}\right), \\
\tilde{A}^{(31)}=\left(\begin{array}{ccc}
0.9 & 0.7 & 0.6 \\
0.7 & 0.9 & 0.6 \\
1 & 0.8 & 0.9
\end{array}\right), & \tilde{A}^{(32)}=\left(\begin{array}{lll}
0.9 & 0.7 & 0.6 \\
0.7 & 0.9 & 0.6 \\
0.8 & 0.9 & 0.9
\end{array}\right), \\
\tilde{A}^{(33)}=\left(\begin{array}{ccc}
0.9 & 0.7 & 0.6 \\
0.7 & 0.9 & 0.6 \\
0.8 & 0.8 & 1
\end{array}\right) . &
\end{array}
$$

The columns of the matrix $\tilde{C} \in \mathcal{I}(9,9)$ and the right-hand side vector $\tilde{b} \in \mathcal{I}(9)$ are computed blockwise according to (19), as follows.

$$
\begin{gathered}
\tilde{C}^{(11)}=\left(\begin{array}{c}
\tilde{A}^{(11)} \otimes_{L} \tilde{x}^{(1)} \\
\tilde{A}^{(11)} \otimes_{L} \tilde{x}^{(2)} \\
\tilde{A}^{(11)} \otimes_{L} \tilde{x}^{(3)}
\end{array}\right)=\left(\begin{array}{l}
0.9 \\
0.7 \\
0.8 \\
0.7 \\
0.7 \\
0.8 \\
0.7 \\
0.7 \\
0.8
\end{array}\right), \quad \tilde{C}^{(12)}=\left(\begin{array}{c}
\tilde{A}^{(12)} \otimes_{L} \tilde{x}^{(1)} \\
\tilde{A}^{(12)} \otimes_{L} \tilde{x}^{(2)} \\
\tilde{A}^{(12)} \otimes_{L} \tilde{x}^{(3)}
\end{array}\right)=\left(\begin{array}{l}
0.8 \\
0.7 \\
0.8 \\
0.6 \\
0.7 \\
0.8 \\
0.6 \\
0.7 \\
0.8
\end{array}\right), \\
\tilde{C}^{(13)}=\left(\begin{array}{c}
0.8 \\
0.7 \\
0.8 \\
0.7 \\
\tilde{A}^{(13)} \otimes_{L} \tilde{x}^{(1)} \\
\tilde{A}^{(13)} \otimes_{L} \tilde{x}^{(2)} \\
\tilde{A}^{(13)} \otimes_{L} \tilde{x}^{(3)}
\end{array}\right)=\left(\begin{array}{l}
0.8 \\
0.7 \\
0.8 \\
0.7 \\
0.7 \\
0.8
\end{array}\right), \quad \tilde{C}^{(21)}=\left(\begin{array}{c}
\tilde{A}^{(21)} \otimes_{L} \tilde{x}^{(1)} \\
\tilde{A}^{(21)} \otimes_{L} \tilde{x}^{(2)} \\
\tilde{A}^{(21)} \otimes_{L} \tilde{x}^{(3)}
\end{array}\right)=\left(\begin{array}{l}
0.6 \\
0.7 \\
0.8 \\
0.6 \\
0.7 \\
0.8
\end{array}\right), \\
\tilde{C}^{(22)}=\left(\begin{array}{l}
\tilde{A}^{(22)} \otimes_{L} \tilde{x}^{(1)} \\
\tilde{A}^{(22)} \otimes_{L} \tilde{x}^{(2)} \\
\tilde{A}^{(22)} \otimes_{L} \tilde{x}^{(3)}
\end{array}\right)=\left(\begin{array}{l}
0.8 \\
0.7 \\
0.7 \\
0.8 \\
0.6 \\
0.7 \\
0.8 \\
0.6 \\
0.7 \\
0.8
\end{array}\right), \quad \tilde{C}^{(23)}=\left(\begin{array}{c}
\tilde{A}^{(23)} \otimes_{L} \tilde{x}^{(1)} \\
\tilde{A}^{(23)} \otimes_{L} \tilde{x}^{(2)} \\
\tilde{A}^{(23)} \otimes_{L} \tilde{x}^{(3)}
\end{array}\right)=\left(\begin{array}{l}
0.8 \\
0.6 \\
0.7 \\
0.8 \\
0.6 \\
0.7 \\
0.8
\end{array}\right),
\end{gathered}
$$




$$
\begin{aligned}
\tilde{C}^{(31)}=\left(\begin{array}{c}
\tilde{A}^{(31)} \otimes_{L} \tilde{x}^{(1)} \\
\tilde{A}^{(31)} \otimes_{L} \tilde{x}^{(2)} \\
\tilde{A}^{(31)} \otimes_{L} \tilde{x}^{(3)}
\end{array}\right)=\left(\begin{array}{c}
0.8 \\
0.7 \\
0.9 \\
0.6 \\
0.7 \\
0.8 \\
0.6 \\
0.7 \\
0.8
\end{array}\right), \quad \tilde{C}^{(32)}=\left(\begin{array}{c}
\tilde{A}^{(32)} \otimes_{L} \tilde{x}^{(1)} \\
\tilde{A}^{(32)} \otimes_{L} \tilde{x}^{(2)} \\
\tilde{A}^{(32)} \otimes_{L} \tilde{x}^{(3)}
\end{array}\right)=\left(\begin{array}{l}
0.8 \\
0.7 \\
0.8 \\
0.6 \\
0.7 \\
0.8 \\
0.6 \\
0.7 \\
0.8
\end{array}\right), \\
\tilde{C}^{(33)}=\left(\begin{array}{c}
0.8 \\
0.7 \\
0.9 \\
0.6 \\
\tilde{A}^{(33)} \otimes_{L} \tilde{x}^{(1)} \\
\tilde{A}^{(33)} \otimes_{L} \tilde{x}_{L}^{(2)} \tilde{x}^{(3)}
\end{array}\right)=\left(\begin{array}{l}
0.9 \\
0.8 \\
0.9 \\
0.9 \\
0.6 \\
0.7 \\
0.9
\end{array}\right), \tilde{b}=\left(\begin{array}{c}
\tilde{x}^{(1)} \\
\tilde{x}^{(2)} \\
\tilde{x}^{(3)}
\end{array}\right)=\left(\begin{array}{c}
0.7 \\
0.8 \\
0.9 \\
0.7 \\
0.8 \\
0.9
\end{array}\right) .
\end{aligned}
$$

Hence, we wish to recognize the solvability of the system

$$
\tilde{C} \otimes_{L} \alpha=\left(\begin{array}{ccccccccc}
0.9 & 0.8 & 0.8 & 0.8 & 0.8 & 0.8 & 0.8 & 0.8 & 0.8 \\
0.7 & 0.7 & 0.7 & 0.7 & 0.7 & 0.7 & 0.7 & 0.7 & 0.7 \\
0.8 & 0.8 & 0.8 & 0.8 & 0.8 & 0.8 & 0.9 & 0.8 & 0.9 \\
0.7 & 0.6 & 0.7 & 0.6 & 0.6 & 0.6 & 0.6 & 0.6 & 0.6 \\
0.7 & 0.7 & 0.7 & 0.7 & 0.7 & 0.7 & 0.7 & 0.7 & 0.7 \\
0.8 & 0.8 & 0.8 & 0.8 & 0.8 & 0.8 & 0.8 & 0.8 & 0.9 \\
0.7 & 0.6 & 0.7 & 0.6 & 0.6 & 0.6 & 0.6 & 0.6 & 0.6 \\
0.7 & 0.7 & 0.7 & 0.7 & 0.7 & 0.7 & 0.7 & 0.7 & 0.7 \\
0.8 & 0.8 & 0.8 & 0.8 & 0.8 & 0.8 & 0.8 & 0.8 & 0.9
\end{array}\right) \otimes_{L}\left(\begin{array}{c}
\alpha_{1} \\
\alpha_{2} \\
\alpha_{3} \\
\alpha_{4} \\
\alpha_{5} \\
\alpha_{6} \\
\alpha_{7} \\
\alpha_{8} \\
\alpha_{9}
\end{array}\right)=\lambda \otimes_{L}\left(\begin{array}{c}
0.9 \\
0.8 \\
0.9 \\
0.7 \\
0.8 \\
0.9 \\
0.7 \\
0.8 \\
0.9
\end{array}\right)=\lambda \otimes_{L} \tilde{b}
$$

The problem is a particular case of the bounded parametric solvability problem, with dimension $n^{2} \times n^{2}$, and can be solved by the algorithm suggested in [28] (see also a brief description in this paper, before Theorem 6).

Depending on the permuted entries of $\tilde{b}$, we distinguish the following four cases: $(a) \lambda \in(0.3,1\rangle,(b) \lambda \in$ $(0.1,0.2), \lambda \in(0.2,0.3\rangle$ and (c) $\lambda \in(0,0.1\rangle$. We can verify that for $\lambda=0.9$ the system $\tilde{C} \otimes_{L} \alpha=\lambda \otimes_{L} \tilde{b}$ has a solution $\alpha=(0.9,0.7,0.9,0.8,0.9,0.9,0.8,0.8,1)^{T}$ fulfilling the inequalities $\underline{a}_{i j}-\bar{a}_{i j}+1 \leq \alpha_{(i j)} \leq 1$, for every $(i, j) \in N \times N$.

Using the coefficients $\alpha_{(i j)}$ we get, by Theorem 5, that $\mathbf{X}$ is a strongly tolerable eigenvector of $\mathbf{A}$, with certificate

$$
A=\bigoplus_{(i j) \in N \times N} \alpha_{(i j)} \otimes_{L} \tilde{A}^{(i j)}=\left(\begin{array}{ccc}
0.9 & 0.7 & 0.7 \\
0.7 & 0.9 & 0.7 \\
0.8 & 0.8 & 1
\end{array}\right) .
$$

Example 5. (Numerical illustration: Computing a certificate - no certificate exists)

We assume the same lower and upper bounds for $A \in[\underline{A}, \bar{A}]$ as in Example 4 and take different bounds for $x \in[\underline{x}, \bar{x}]$.

$$
\underline{A}=\left(\begin{array}{ccc}
0.9 & 0.7 & 0.6 \\
0.7 & 0.9 & 0.6 \\
0.8 & 0.8 & 0.9
\end{array}\right), \quad \bar{A}=\left(\begin{array}{ccc}
1 & 0.8 & 0.8 \\
0.7 & 0.9 & 0.8 \\
1 & 0.9 & 1
\end{array}\right)
$$




$$
\underline{x}=\left(\begin{array}{c}
0.7 \\
0.7 \\
0.7
\end{array}\right), \quad \bar{x}=\left(\begin{array}{l}
1 \\
1 \\
1
\end{array}\right) .
$$

Then the generators of A stay the same and

$$
\tilde{x}^{(1)}=\left(\begin{array}{c}
1 \\
0.7 \\
0.7
\end{array}\right), \quad \tilde{x}^{(2)}=\left(\begin{array}{c}
0.7 \\
1 \\
0.7
\end{array}\right), \quad \tilde{x}^{(3)}=\left(\begin{array}{c}
0.7 \\
0.7 \\
1
\end{array}\right) .
$$

The matrix $\tilde{C}$ and the right-hand side $\tilde{b}$ then are

$$
\tilde{C}=\left(\begin{array}{ccccccccc}
1 & 0.9 & 0.9 & 0.9 & 0.9 & 0.9 & 0.9 & 0.9 & 0.9 \\
0.7 & 0.7 & 0.7 & 0.7 & 0.7 & 0.7 & 0.7 & 0.7 & 0.7 \\
0.8 & 0.8 & 0.8 & 0.8 & 0.8 & 0.8 & 1 & 0.8 & 0.8 \\
0.7 & 0.8 & 0.7 & 0.7 & 0.7 & 0.7 & 0.7 & 0.7 & 0.7 \\
0.9 & 0.9 & 0.9 & 0.9 & 0.9 & 0.9 & 0.9 & 0.9 & 0.9 \\
0.8 & 0.8 & 0.8 & 0.8 & 0.8 & 0.8 & 0.8 & 0.9 & 0.8 \\
0.7 & 0.6 & 0.8 & 0.6 & 0.6 & 0.6 & 0.6 & 0.6 & 0.6 \\
0.6 & 0.6 & 0.6 & 0.6 & 0.6 & 0.8 & 0.6 & 0.6 & 0.6 \\
0.9 & 0.9 & 0.9 & 0.9 & 0.9 & 0.9 & 0.9 & 0.9 & 1
\end{array}\right), \tilde{b}=\left(\begin{array}{c}
1 \\
0.7 \\
0.7 \\
0.7 \\
1 \\
0.7 \\
0.7 \\
0.7 \\
1
\end{array}\right) .
$$

Similarly as in the previous example we distinguish, depending on the permuted entries of $\tilde{b}$, the following three cases: (a) $\lambda \in(0.3,1\rangle$, (b) $\lambda \in(0,0.3\rangle$ and (c) $\lambda=0$. It can be verified that the system $\tilde{C} \otimes_{L} \alpha=\lambda \otimes_{L} \tilde{b}$ has no solution in any of these cases.

Consequently, the considered system is not solvable for any value of $\lambda$. That is, no certificate for strong tolerability exists and the given $\mathbf{X}$ is not a strongly tolerable eigenvector of $\mathbf{A}$.

\section{Strongly Universal Interval Eigenvectors in a Max-Łuk Algebra}

In this section, we present two necessary and sufficient conditions for characterizing a strongly universal eigenvector. The first condition is based on the generators of $\mathbf{A}$, while the second one uses the lower bound and the upper bound of $\mathbf{A}$.

Theorem 7. Let $\mathbf{A}$ and $\mathbf{X}$ be given such that $\underline{a}_{i j}=\bar{a}_{i j}$ for some $i, j \in N$. The interval vector $\mathbf{X}$ is a strongly universal max- $\succeq$ uk eigenvector of the interval matrix $\mathbf{A}=[\underline{A}, \bar{A}]$ if and only if there exists an $x \in \mathbf{X}$ and $a$ $\lambda \in \mathcal{I}$ such that

$$
\tilde{A}^{(i j)} \otimes_{L} x=\lambda \otimes_{L} x \quad \text { for every } i, j \in N .
$$

Proof. Let us assume that there are $x \in \mathbf{X}$ and $\lambda \in \mathcal{I}$ fulfilling condition (30). If $A \in \mathcal{I}\left(n^{2}\right)$ is an arbitrary matrix in $\mathbf{A}$, then $A$ is a max-Łuk linear combination $A=\bigoplus_{i j \in N} \alpha_{i j} \otimes_{L} \tilde{A}^{(i j)}$ for some coefficients $\alpha_{i j} \in \mathcal{I}, i, j \in N$ with $\underline{a}_{i j}-\bar{a}_{i j}+1 \leq \alpha_{i j} \leq 1$. According to Lemma 1(ii),

$$
\begin{aligned}
A \otimes_{L} x & =\left(\bigoplus_{i j \in N} \alpha_{i j} \otimes_{L} \tilde{A}^{(i j)}\right) \otimes_{L} x=\bigoplus_{i j \in N} \alpha_{i j} \otimes_{L}\left(\tilde{A}^{(i j)} \otimes_{L} x\right) \\
& =\bigoplus_{i j \in N} \alpha_{i j} \otimes_{L}\left(\lambda \otimes_{L} x\right)=\left(\bigoplus_{i j \in N} \alpha_{i j} \otimes_{L} \lambda\right) \otimes_{L} x=\lambda \otimes_{L} x
\end{aligned}
$$

because $\underline{a}_{i j}=\bar{a}_{i j}$ for some $i, j \in N$ implies $\bigoplus_{i j \in N} \alpha_{i j}=1$ and $\bigoplus_{i j \in N} \alpha_{i j} \otimes_{L} \lambda=\lambda$. By Definition 1 , $\mathbf{X}$ is a strongly universal eigenvector of $\mathbf{A}$. The converse implication follows immediately. 
Theorem 8. Suppose given an interval matrix $\mathbf{A}=[\underline{A}, \bar{A}]$ and interval vector $\mathbf{X}$ with bounds $\underline{x}, \bar{x}$. Then $\mathbf{X}$ is a strongly universal eigenvector of $\mathbf{A}$ if and only if there are $\lambda \in \mathcal{I}$ and $x \in \mathbf{X}$ such that $\underline{A} \otimes_{L} x=\lambda \otimes_{L} x$ and $\bar{A} \otimes_{L} x=\lambda \otimes_{L} x$.

Proof. Let us suppose that there are $\lambda$ and $x \in \mathbf{X}$ such that $\underline{A} \otimes_{L} x=\bar{A} \otimes x=\lambda \otimes x$. From the monotonicity of the operations $\oplus$ and $\otimes_{L}$ we get $\lambda \otimes_{L} x=\underline{A} \otimes_{L} x \leq A \otimes_{L} x \leq \bar{A} \otimes_{L} x=\lambda \otimes_{L} x$ for every $A \in \mathbf{A}$. The converse implication is trivial.

The condition described in Theorem 8 can be verified by solving a two-sided max-Łuk system defined as follows. Define the block matrices $C \in \mathcal{I}(2 n, n), D \in \mathcal{I}(2 n, n)$ by

$$
C=\left(\begin{array}{c}
\underline{A} \otimes_{L} \tilde{x}^{(1)} \ldots \underline{A} \otimes_{L} \tilde{x}^{(n)} \\
\bar{A} \otimes_{L} \tilde{x}^{(1)} \ldots \bar{A} \otimes_{L} \tilde{x}^{(n)}
\end{array}\right), \quad D=\left(\begin{array}{ccc}
\tilde{x}^{(1)} & \ldots & \tilde{x}^{(n)} \\
\tilde{x}^{(1)} & \ldots & \tilde{x}^{(n)}
\end{array}\right) .
$$

Theorem 9. Assume that an interval matrix $\mathbf{A}=[\underline{A}, \bar{A}]$ and an interval vector $\mathbf{X}=[\underline{x}, \bar{x}]$ are given. Then $\mathbf{X}$ is a strongly universal eigenvector of $\mathbf{A}$ if and only if the bounded two-sided max- $\succeq$ uk linear system with variable $\beta \in \mathcal{I}(n)$

$$
\begin{aligned}
C \otimes_{L} \beta & =\lambda \otimes_{L} D \otimes_{L} \beta \\
\underline{x}-\bar{x}+1 & \leq \beta \leq 1
\end{aligned}
$$

is solvable for some value of the parameter $\lambda \in \mathcal{I}$. If $\beta \in \mathcal{I}(n)$ is a solution to the system, then $x=\bigoplus_{i=1}^{n} \beta_{i} \otimes \tilde{x}^{i}$ satisfies the condition in Theorem 8.

Proof. Let us suppose that there is a $\lambda$ such that the two-sided system $C \otimes \beta=\lambda \otimes D \otimes \beta$ has a solution $\beta$. Put $x=\bigoplus_{i=1}^{n} \beta_{i} \otimes \tilde{x}^{i}$. Then the following formulas are equivalent

$$
\begin{gathered}
C \otimes \beta=\lambda \otimes D \otimes \beta \\
\bigoplus_{i=1}^{n} \underline{A} \otimes \tilde{x}^{(i)} \otimes \beta_{i}=\lambda \otimes \bigoplus_{i=1}^{n} \tilde{x}^{(i)} \otimes \beta_{i} \text { and } \bigoplus_{i=1}^{n} \bar{A} \otimes \tilde{x}^{(i)} \otimes \beta_{i}=\lambda \otimes \bigoplus_{i=1}^{n} \tilde{x}^{(i)} \otimes \beta_{i} \\
\bigoplus_{i=1}^{n} \underline{A} \otimes \tilde{x}^{(i)} \otimes \beta_{i}=\lambda \otimes \bigoplus_{i=1}^{n} \tilde{x}^{(i)} \otimes \beta_{i} \text { and } \bigoplus_{i=1}^{n} \bar{A} \otimes \tilde{x}^{(i)} \otimes \beta_{i}=\lambda \otimes \bigoplus_{i=1}^{n} \tilde{x}^{(i)} \otimes \beta_{i} \\
\underline{A} \otimes \bigoplus_{i=1}^{n} \beta_{i} \otimes \tilde{x}^{(i)}=\lambda \otimes \bigoplus_{i=1}^{n} \beta_{i} \otimes \tilde{x}^{(i)} \text { and } \bar{A} \otimes \bigoplus_{i=1}^{n} \beta_{i} \otimes \tilde{x}^{(i)}=\lambda \otimes \bigoplus_{i=1}^{n} \beta_{i} \otimes \tilde{x}^{(i)} \\
\underline{A} \otimes x=\lambda \otimes x \text { and } \bar{A} \otimes x=\lambda \otimes x .
\end{gathered}
$$

The assertion follows by Theorem 8 .

By Theorem 9, the verification of whether $\mathbf{X}$ is a strongly universal eigenvector of $\mathbf{A}$ is reduced to the verification of the solvability of the system $C \otimes_{L} x=\lambda \otimes_{L} D \otimes_{L} x$. A similar situation in max-min algebra is solved by a polynomial algorithm for the solvability of such a system, with a complexity equal to $O\left(n^{3}\right)$ [29].

In max-plus algebra, the solvability of the considered system has been generally shown to be polynomially equivalent to solving a mean-payoff game [30]. That is, there exist efficient pseudopolynomial algorithms for this problem. On the other hand, the existence of a polynomial algorithm is a long-standing open question. Similarly, for a max-Łuk algebra, the existence of a polynomial algorithm for the solvability recognition problem remains open.

Example 6. (Numerical illustration: Strongly universal interval eigenvector) 
Assume that $A \in[\underline{A}, \bar{A}]$ and $x \in[\underline{x}, \bar{x}]$

$$
\begin{array}{cc}
\underline{A}=\left(\begin{array}{ccc}
0.6 & 0.3 & 0.4 \\
0.1 & 0 & 0.2 \\
0.2 & 0.2 & 0.6
\end{array}\right), \quad \bar{A}=\left(\begin{array}{lll}
0.6 & 0.7 & 0.5 \\
0.4 & 0.6 & 0.5 \\
0.5 & 0.7 & 0.6
\end{array}\right) \\
\underline{x}=\left(\begin{array}{l}
0.1 \\
0.2 \\
0.1
\end{array}\right), \quad \bar{x}=\left(\begin{array}{l}
0.7 \\
0.6 \\
0.7
\end{array}\right) .
\end{array}
$$

It is easy to verify that for a given $\lambda=0.4, x=(0.1,0.2,0.1)^{T}$ and for every matrix generator $\tilde{A}^{i j}, i, j \in N$,

$$
\tilde{A}^{i j} \otimes x=\tilde{A}^{i j} \otimes\left(\begin{array}{l}
0.1 \\
0.2 \\
0.1
\end{array}\right)=\left(\begin{array}{l}
0 \\
0 \\
0
\end{array}\right)=0.4 \otimes\left(\begin{array}{l}
0.1 \\
0.2 \\
0.1
\end{array}\right)=\lambda \otimes_{L} x .
$$

Hence, in view of Theorem 7 , the interval vector $\mathbf{X}$ is a strongly universal max- \pm uk eigenvector of the interval matrix $\mathbf{A}$.

Theorem 9 offers a more systematic approach: find a solution to the two-sided system (32) and (33) with unknown coefficients $\beta$, for some $\lambda^{\prime} \in \mathcal{I}$ :

$$
\begin{gathered}
\left(\begin{array}{ccc}
0.3 & 0 & 0.1 \\
0 & 0 & 0 \\
0 & 0 & 0.3 \\
0.3 & 0.3 & 0.2 \\
0.1 & 0.2 & 0.2 \\
0.2 & 0.3 & 0.3
\end{array}\right) \otimes_{L}\left(\begin{array}{l}
\beta_{1} \\
\beta_{2} \\
\beta_{3}
\end{array}\right)=\lambda^{\prime} \otimes_{L}\left(\begin{array}{lll}
0.7 & 0.1 & 0.1 \\
0.2 & 0.6 & 0.2 \\
0.1 & 0.1 & 0.7 \\
0.7 & 0.1 & 0.1 \\
0.2 & 0.6 & 0.2 \\
0.1 & 0.1 & 0.7
\end{array}\right) \otimes_{L}\left(\begin{array}{l}
\beta_{1} \\
\beta_{2} \\
\beta_{3}
\end{array}\right), \\
\left(\begin{array}{l}
0.4 \\
0.6 \\
0.4
\end{array}\right) \leq\left(\begin{array}{l}
\beta_{1} \\
\beta_{2} \\
\beta_{3}
\end{array}\right) \leq\left(\begin{array}{l}
1 \\
1 \\
1
\end{array}\right) .
\end{gathered}
$$

In this particular instance, it is easy to verify that $\beta^{\prime}=(0.9,0.8,0.8)^{T}$ is a solution to the system with $\lambda^{\prime}=0.6$. Then the corresponding linear combination of generators, $x^{\prime}=\left(0.9 \otimes_{L} \tilde{x}^{(1)}\right) \oplus\left(0.8 \otimes_{L} \tilde{x}^{(2)}\right) \oplus$ $\left(0.8 \otimes_{L} \tilde{x}^{(3)}\right)=(0.6,0.4,0.5)^{T}$, satisfies the condition from Theorem 8. It is worth noticing that we have found two different universal eigenvectors $x$ and $x^{\prime}$ for two different values $\lambda$ and $\lambda^{\prime}$ in this example. Hence, we have shown that neither the "strongly universal" eigenvalue nor the strongly universal eigenvector are uniquely determined.

\section{Conclusions}

Strong versions of the notion of an interval eigenvector of an interval matrix in a max- $\succeq u k$ algebra have been investigated in this paper. The steady states of a given discrete events system (DES) correspond to eigenvectors of the transition matrix of the system under consideration. When the entries of the state vectors and transition matrix are supposed to be contained in some intervals, then several types of interval eigenvector can be defined, according to the choice of the quantifiers used in the definition. Three of the main important types of interval eigenvectors of a given interval matrix in a max-Łuk algebra have been studied: the strong eigenvector, the strongly tolerable eigenvector, and the strongly universal eigenvector.

Using vector generators and matrix generators belonging to given intervals, the structure of the eigenspace for each of the above mentioned types has been described, and necessary and sufficient conditions for the existence of an interval eigenvector have been formulated. Moreover, recognition 
algorithms have been suggested for the recognition of these conditions for the first two types: strong and strongly tolerable eigenvector. The existence of an efficient recognition algorithm for the strongly universal type has not been shown. This question remains as a challenge for future research.

These results can be useful in practical applications aimed at the construction of real DES working with Łukasiewicz fuzzy logic. The results have been illustrated by numerical examples.

Author Contributions: Investigation, M.G., Z.N. and J.P.; Writing-review and editing, M.G., Z.N. and J.P. All authors contributed equally to this manuscript. All authors have read and agreed to the published version of the manuscript.

Funding: This research was funded by the Czech Science Foundation (GAČR) \#18-01246S and by the Faculty of Informatics and Management UHK, specific research project 2107 Computer Networks for Cloud, Distributed Computing, and Internet of Things III.

Conflicts of Interest: The authors declare no conflict of interest. The funders had no role in the design of the study; in the collection, analyses, or interpretation of data; in the writing of the manuscript, or in the decision to publish the results.

\section{References}

1. Schweizer, B.; Sklar, A. Statistical metric spaces. Pac. J. Math. 1960, 10, 313-334. [CrossRef]

2. Gottwald, S. A Treatise on Many-Valued Logics; Studies in Logic and Computation, Research Studies Press: Baldock, Great Britain, 2001.

3. Gavalec, M. Periodicity in Extremal Algebras; Gaudeamus: Hradec Králové, Czech Republic, 2004.

4. Golan, J.S. Semirings and Their Applications; Springer: Berlin, Germany, 2013.

5. Gondran, M.; Minoux, M. Graphs, Dioids and Semirings: New Models and Algorithms; Springer: Berlin, Germany, 2008; Volume 41.

6. Kolokoltsov, V.; Maslov, V.P. Idempotent Analysis and Its Applications; Springer: Berlin, Germany, 1997; Volume 401.

7. Mysková, H. Interval eigenvectors of circulant matrices in fuzzy algebra. Acta Electrotech. Inform. 2012, $12,57$. [CrossRef]

8. Mysková, H. Weak stability of interval orbits of circulant matrices in fuzzy algebra. Acta Electrotech. Inform. 2012, 12, 51. [CrossRef]

9. Plavka, J. On the weak robustness of fuzzy matrices. Kybernetika 2013, 49, 128-140.

10. Tan, Y.J. Eigenvalues and eigenvectors for matrices over distributive lattices. Linear Algebra Appl. 1998, 283, 257-272. [CrossRef]

11. Zimmermann, K. Extremální Algebra; Útvar vědeckỳch Informací Ekonomického Ústavu ČSAV: Praha, Czech Republic, 1976.

12. Saleem, N.; Abbas, M.; De la Sen, M. Optimal Approximate Solution of Coincidence Point Equations in Fuzzy Metric Spaces. Mathematics 2019, 7, 327. [CrossRef]

13. Alolaiyan, H.; Saleem, N.; Abbas, M. A natural selection of a graphic contraction transformation in fuzzy metric spaces. J. Nonlinear Sci. Appl. 2018, 11, 218-227. [CrossRef]

14. Butkovic, P. Max-Linear Systems: Theory and Algorithms; Springer: Berlin, Germany, 2010; p. 272. [CrossRef]

15. Cuninghame-Green, R.A. Minimax Algebra; Springer: Berlin, Germany, 2012; Volume 166.

16. Gavalec, M. Monotone eigenspace structure in max-min algebra. Linear Algebra Appl. 2002, 345, $149-167$. [CrossRef]

17. Gavalec, M.; Němcová, Z.; Sergeev, S. Tropical linear algebra with the Łukasiewicz T-norm. Fuzzy Sets Syst. 2015, 276, 131-148. [CrossRef]

18. Gavalec, M.; Rashid, I.; Cimler, R. Eigenspace structure of a max-drast fuzzy matrix. Fuzzy Sets Syst. 2014, 249, 100-113. [CrossRef]

19. Rashid, I.; Gavalec, M.; Cimler, R. Eigenspace structure of a max-prod fuzzy matrix. Fuzzy Sets Syst. 2016, 303, 136-148. [CrossRef]

20. Collins, P.; Niqui, M.; Revol, N. A validated real function calculus. Math. Comput. Sci. 2011, 5, 437-467. [CrossRef]

21. Fiedler, M.; Nedoma, J.; Ramík, J.; Rohn, J.; Zimmermann, K. Linear Optimization Problems with Inexact Data; Springer: Berlin, Germany, 2006. 
22. Gavalec, M.; Ramík, J.; Zimmermann, K. Interval eigenproblem in max-min algebra. In Decision Making and Optimization; Springer: Berlin, Germany, 2015; pp. 163-181.

23. Litvinov, G.L.; Sobolevskī̄, A.N. Idempotent interval analysis and optimization problems. Reliab. Comput. 2001, 7, 353-377. [CrossRef]

24. Gavalec, M.; Plavka, J. Monotone interval eigenproblem in max-min algebra. Kybernetika 2010, 46, 387-396.

25. Gavalec, M.; Plavka, J.; Tomášková, H. Interval eigenproblem in max-min algebra. Liner Algebra Appl. 2014, 440, 24-33. [CrossRef]

26. Gavalec, M.; Plavka, J.; Ponce, D. Tolerance types of interval eigenvectors in max-plus algebra. Inf. Sci. 2017, 367, 14-27. [CrossRef]

27. Gavalec, M.; Zimmermann, K. Classification of solutions to systems of two-sided equations with interval coefficients. Int. J. Pure Appl. Math. 2008, 45, 533.

28. Gavalec, M.; Němcová, Z. Solvability of a Bounded Parametric System in Max-Łukasiewicz Algebra. Mathematics 2020, 8, 1026. [CrossRef]

29. Gavalec, M.; Zimmermann, K. Solving systems of two sided (max,min) linear equations. Kybernetika 2010, $46,405-414$.

30. Allamigeon, X.; Fahrenberg, U.; Gaubert, S.; Katz, R.D.; Legay, A. Tropical Fourier-Motzkin elimination, with an application to real-time verification. Int. J. Algebra Comput. 2014, 24, 569-607. [CrossRef]

(c) 2020 by the authors. Licensee MDPI, Basel, Switzerland. This article is an open access article distributed under the terms and conditions of the Creative Commons Attribution (CC BY) license (http://creativecommons.org/licenses/by/4.0/). 